Supporting Information for

\title{
Graphene Field Effect Transistor-based Immunosensor for Ultrasensitive Noncompetitive Detection of Small Antigens
}

Yasushi Kanai, ${ }^{\dagger} \nabla$ Yuki Ohmuro-Matsuyama, ${ }^{\dagger, \nabla}$ Masami Tanioku, ${ }^{\dagger}$ Shota Ushiba, ${ }^{\S}$ Takao Ono, ${ }^{\dagger}$

Koichi Inoue, ${ }^{\dagger}$ Tetsuya Kitaguchi, ${ }^{\ddagger}$ Masahiko Kimura, ${ }^{\S}$ Hiroshi Ueda, ${ }^{\ddagger}, *$ Kazuhiko Matsumoto ${ }^{\dagger}$

$\dagger$ Institute of Scientific and Industrial Research, Osaka University, Ibaraki, Osaka 567-0047, Japan

$\$$ Laboratory for Chemistry and Life Sciences, Institute of Innovative Research, Tokyo Institute of Technology, Nagatsuta-cho, Yokohama, Kanagawa 226-8503, Japan

$\S$ Murata Manufacturing Co. Ltd., Higashikotari, Nagaokakyo-shi, Kyoto 617-8555, Japan

*Phone/Fax: +81-45-924-5248. E-mail: ueda@res.titech.ac.jp

\section{Table of Contents}

SI Materials and Methods

S-2

SI References

S-4

Figure S-1. Antigen-dependent association of $\mathrm{V}_{\mathrm{H}} / \mathrm{V}_{\mathrm{L}}$ confirmed by ELISA

S-5

Figure S-2. OS G-FET IA for BGP-C7 using Trx-BAP-V $\mathrm{V}_{\mathrm{H}}$

S-6

Figure S-3. Transport characteristics of G-FETs prepared for each measurement. 


\section{SI Materials \& Methods}

\section{Materials}

The KOD-pus DNA polymerase was obtained from Toyobo (Osaka, Japan). Restriction enzymes and Escherichia coli SHuffle T7 Express lys Y were from New England Biolabs-Japan (Tokyo, Japan). Oligonucleotides were from Operon-Eurofins (Tokyo, Japan). The Pure Yield plasmid miniprep kit was from Promega (Tokyo, Japan). Calf intestinal alkaline phosphatase (CIAP), Talon metal affinity resin and Talon disposable gravity column were obtained from Takara-Bio (Otsu, Japan). Horseradish peroxidase avidin D was form Vector Laboratories (CA, USA). Immunoblock and human pooled serum were from DS Pharma (Osaka, Japan). Polystylene microplates for ELISA was from Greiner (Tokyo, Japan). TMBZ (3,3',5,5'-Tetramethylbenzidine) was from Dojindo (Kumamoto, Japan). Peptides, BGP-C7

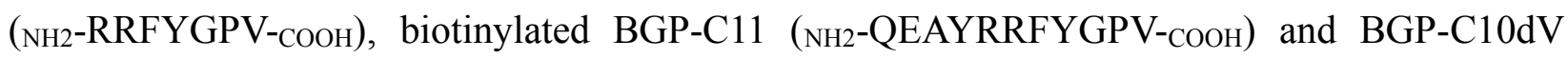
( $\left.\mathrm{NH}_{2}-\mathrm{EAYRRFYGP-}-\mathrm{COOH}\right)$ were from Lifetein (NJ, USA). HRP-conjugated anti-His 6 antibody was from Roche Diagnostics (Tokyo, Japan). Other chemicals and reagents were from Wako Pure Chemicals (Osaka, Japan).

\section{Plasmid constructions}

Maltose-binding protein (MBP)-fused $\mathrm{V}_{\mathrm{L}}$ was constructed previously ${ }^{1}$. To generate thioredoxin (Trx) and biotin acceptor peptide (BAP)-fused $\mathrm{V}_{\mathrm{H}}$, the $\mathrm{V}_{\mathrm{H}}$ fragment was amplified by PCR using primers

VHG4SNotIBack (5'-GAATTCGCGGCCGCTTACTTGGTGGAAGCGCT-3') and VHstopNotIFor (5'-GAATTCGCGGCCGCCGGTGGTGGTGGTAGCCAAGTAAAGCTGCAGCAG-3'), with an affinity-matured $\mathrm{V}_{\mathrm{H}}$ fragment for osteocalcin $\mathrm{R} 4 \mathrm{~A} 10^{2}$ as a template (restriction site underlined). The amplified fragment was digested with NotI, and inserted to a pET32-based expression vector for BAP-fused luciferase ${ }^{3}$, which had been digested with NotI and treated with CIAP, to yield pET32-BAP-VH(R4A10). To generate APP tag-fused $\mathrm{V}_{\mathrm{H}}$, the $\mathrm{V}_{\mathrm{H}}$ fragment was amplified by PCR using the primers NcoIVHBack (5'-AAGGCCATGGAGGTACAGCTGGAGGAGTC-3') and HindIIIStopVHFor (5'-GTGTAAGCTTTTAGCCACCACCGGCCGCGCTC-3') with BAP-fused $V_{H}$ constructed above as a template. The amplified fragment and pRsetA carrying hyperacidic module derived from murine amyloid precursor protein (APP) ${ }^{4}$ were both digested with $\mathrm{NcoI}$ and HindIII, and the 
amplified VH fragment was inserted between NcoI and HindIII sites of pRsetA-APP to generate pRsetA-APP-VH(R4A10).

\section{Expression and purification of MBP-fused $V_{L}$, biotinylated $V_{H}$ and APP tag-fused $V_{H}$}

The fusion proteins were expressed in Escherichia coli SHuffle T7 Express lys Y as a His6-tagged protein. To purify the expressed fusion protein, Talon metal affinity resin was used according to the manufacturer's instruction. The biotinylation of Trx-BAP- $\mathrm{V}_{\mathrm{H}}$ was performed in the same way as previously described ${ }^{3}$.

\section{ELISA}

To confirm the binding of $M B P-V_{L}$ and $\operatorname{Trx}-B A P-V_{H}$ in the presence of the antigen, a microplate well was coated with MBP-V $\mathrm{V}_{\mathrm{L}}(1 \mu \mathrm{g} / \mathrm{mL})$ at $4^{\circ} \mathrm{C}$ overnight, washed thrice with PBST and blocked with 20\% Immunoblock at room temperature for $1 \mathrm{~h}$. After three washes with PBST, varied concentration of BGP-C7 and Trx-BAP-V $\mathrm{V}_{\mathrm{H}}(1 \mu \mathrm{g} / \mathrm{mL})$ were mixed in $5 \%$ Immunoblock/PBST, and reacted at room temperature for $1 \mathrm{~h}$. After three washes with PBST, horseradish peroxidase (HRP)-avidin D (1/10,000 in 5\% Immunoblock/PBST) was applied and incubated for $30 \mathrm{~min}$ at room temperature. After three washes with PBST, the development was performed with $100 \mu \mathrm{L}$ TMBZ solution $\left(100 \mu \mathrm{g} / \mathrm{mL}\right.$ TMBZ and $0.04 \mu \mathrm{L} / \mathrm{mL} \mathrm{H}_{2} \mathrm{O}_{2}$ in $100 \mathrm{mM}$ NaOAc, pH 6.0).

To confirm the antigen binding activity of $\mathrm{MBP}-\mathrm{V}_{\mathrm{L}}$ and $\mathrm{APP}-\mathrm{V}_{\mathrm{H}}$, a microplate well was coated with streptavidin $(10 \mu \mathrm{g} / \mathrm{mL})$ at $4^{\circ} \mathrm{C}$ overnight and washed with PBST thrice and blocked with $20 \%$ Immunoblock at room temperature for $1 \mathrm{~h}$. After three washes with PBST, streptavidin was reacted with/without $1 \mu \mathrm{g} / \mathrm{mL}$ biotinylated BGP-C11 at room temperature for $1 \mathrm{~h}$. After washing thrice with PBST, either MBP- $\mathrm{V}_{\mathrm{L}}$ alone, APP- $\mathrm{V}_{\mathrm{H}}$ alone, or the mixture of MBP- $\mathrm{V}_{\mathrm{L}}$ and APP- $\mathrm{V}_{\mathrm{H}}(1 \mu \mathrm{g} / \mathrm{mL}$ each) was applied and incubated at room temperature at $1 \mathrm{~h}$. After three washes with PBST, bound His-tagged proteins were probed with HRP-conjugated anti-His 6 antibody $(1 / 10,000$ in 5\% Immunoblock/PBST) for $30 \mathrm{~min}$. After three washes with PBST, the development was performed with $100 \mu \mathrm{L}$ TMBZ solution. The absorbance was measured with SH-1000 microplate reader (Corona Electric, Ibaraki, Japan). The data obtained was analyzed using a Microsoft Excel software. 


\section{Construction and use of G-FET}

In the constructed G-FET biosensor (Figure 2), source and drain electrodes consisting of $\mathrm{Ti} / \mathrm{Au}$ $(10 \mathrm{~nm} / 30 \mathrm{~nm})$ were fabricated on a $\mathrm{SiO}_{2}$ substrate by conventional photolithography and lift-off process. Graphene was synthesized on copper foils by a chemical vapor deposition method ${ }^{5}$. Synthesized graphene was transferred on the substrate on a chip. G-FETs were immobilized using $1 \mathrm{mM}$ 1-pyrenebutanoic acid succinimidyl ester (PBASE) in 2-methoxyethanol for $1 \mathrm{~h}$. The pyrenyl group of PBASE strongly bound to the graphene surface via $\pi-\pi$ stacking and is used as a linker ${ }^{6}$. After the PBASE immobilization, G-FETs were sequentially washed with 2-methoxyethanol and $10 \mathrm{mM}$ phosphate buffer ( $\mathrm{PB}, \mathrm{pH}$ 7.0), and immersed for $18 \mathrm{~h}$ in $\mathrm{PB}$

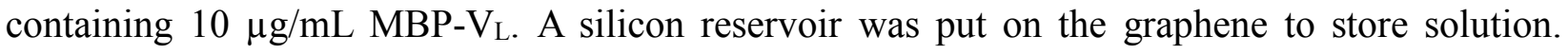
Electrical measurements were carried out with $10 \mathrm{mM}$ PB and $1 \mu \mathrm{g} / \mathrm{mL}$ of Trx-BAP-V $V_{\mathrm{H}}$ or $\mathrm{APP}-\mathrm{V}_{\mathrm{H}}$ at $\mathrm{pH}$ 7.0, with varied amounts of antigen or non-antigen peptide. The average signal and standard deviation (SD) during the period 1 min after the addition of a sample to next sample addition was plotted. $\mathrm{Ag} / \mathrm{AgCl}$ reference electrode was used as the top gate electrode.

\section{SI References}

1. Lim, S. L.; Ichinose, H.; Shinoda, T.; Ueda, H., Noncompetitive detection of low molecular weight peptides by open sandwich immunoassay. Anal. Chem. 2007, 79 (16), 6193-6200.

2. Iwai, H.; Oztürk, B.; Ihara, M.; Ueda, H., Antibody affinity maturation in vitro using unconjugated peptide antigen. Protein Eng. Des. Sel. 2010, 23 (4), 185-193.

3. Ohmuro-Matsuyama, Y.; Yamashita, T.; Gomi, K.; Yamaji, H.; Ueda, H., Evaluation of protein-ligand interactions using the luminescent interaction assay FlimPIA with streptavidin-biotin linkage. Anal. Biochem. 2018, 563, 61-66.

4. Wongso, D.; Dong, J.; Ueda, H.; Kitaguchi, T., Flashbody: a next generation Fluobody with fluorescence intensity enhanced by antigen binding. Anal. Chem. 2017, 89 (12), 6719-6725.

5. Li, X.; Cai, W.; An, J.; Kim, S.; Nah, J.; Yang, D.; Piner, R.; Velamakanni, A.; Jung, I.; Tutuc, E.; Banerjee, S. K.; Colombo, L.; Ruoff, R. S., Large-area synthesis of high-quality and uniform graphene films on copper foils. Science 2009, 324 (5932), 1312-1314.

6. Ohno, Y.; Maehashi, K.; Inoue, K.; Matsumoto, K., Label-free aptamer-based immunoglobulin sensors using graphene field-effect transistors. Jpn. J. Appl. Phys. 2011, 50 (070120). doi: 10.7567/JJAP.57.070120 
A

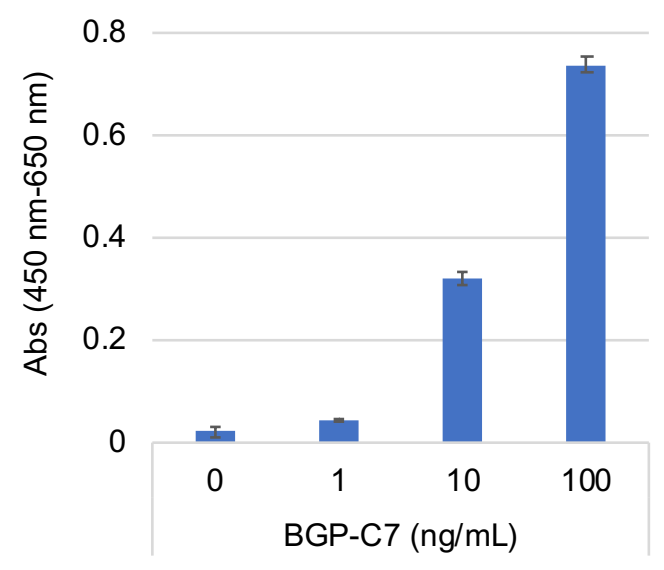

B

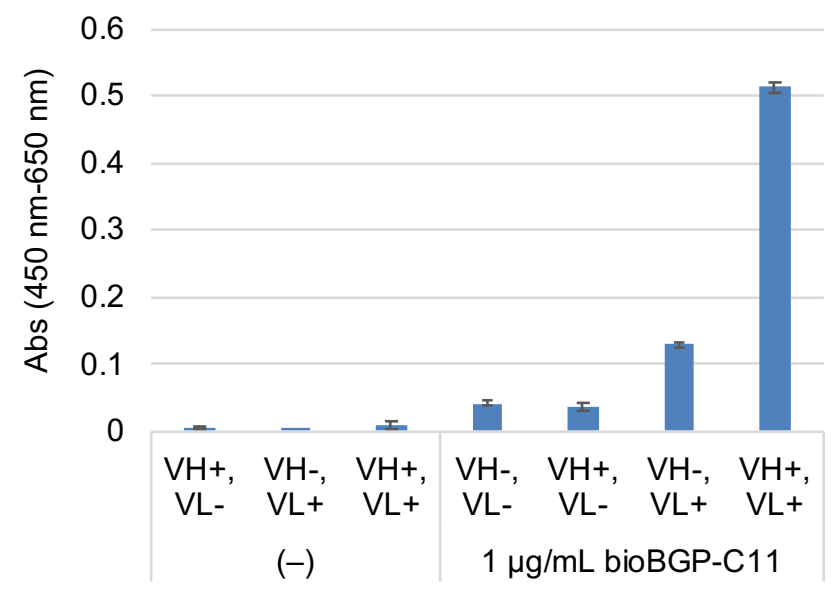

Figure S1. Antigen-dependent association of $V_{H} / V_{L}$ fragments confirmed by ELISA. (A) Open sandwich ELISA using Trx-BAP- $V_{H}$ and immobilized MBP- $V_{L}$. The well-bound Trx-BAP- $\mathrm{V}_{\mathrm{H}}$ was detected with Avidin-HRP. (B) Antigen binding ELISA using APP- $\mathrm{V}_{\mathrm{H}}$ and $\mathrm{MBP}-\mathrm{V}_{\mathrm{L}}$. The proteins were detected with HRP-labeled anti-His 6 antibody. Average of three measurements with an error bar for $1 \mathrm{SD}$ is shown. 
A

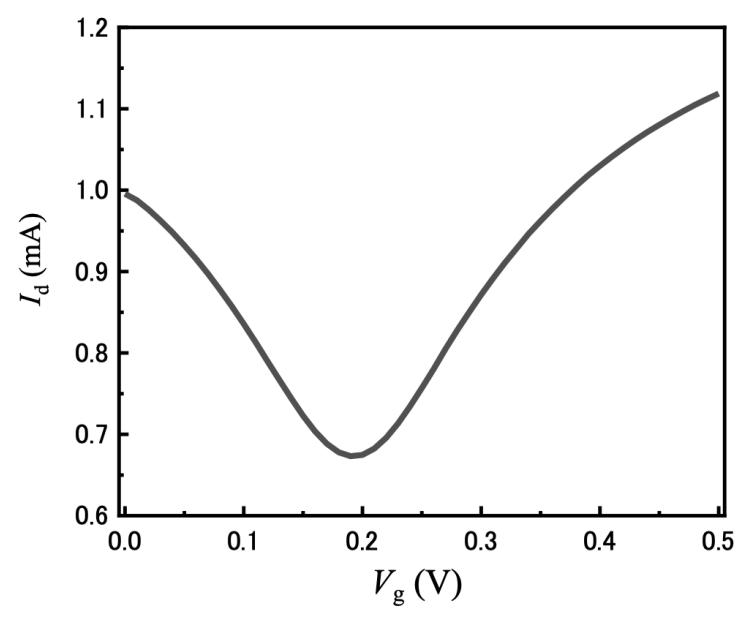

B

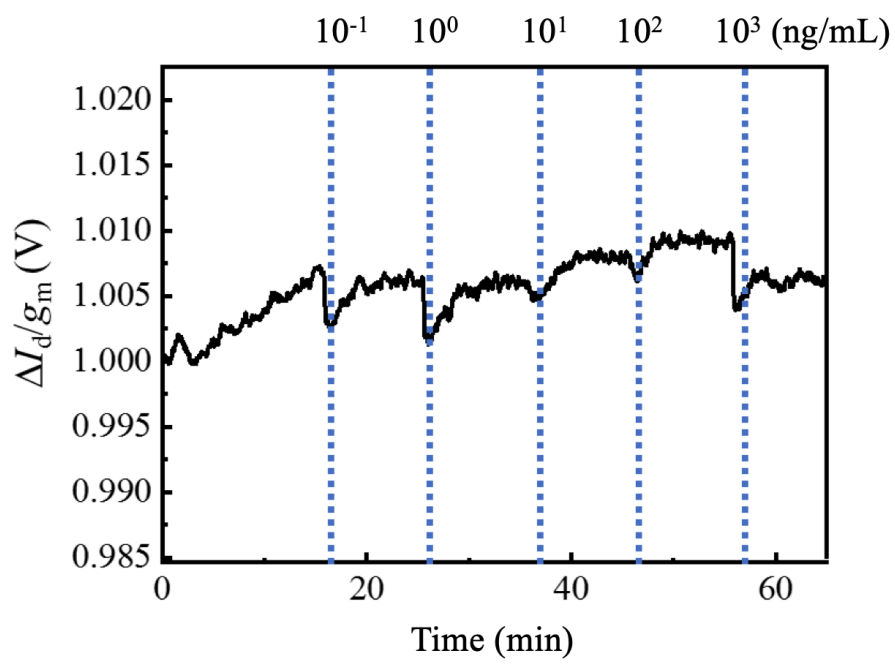

Figure S2. OS G-FET IA for BGP-C7 using Trx-BAP-V $\mathbf{H}_{\mathbf{H}}$ (A) Transport characteristics of the G-FET. (B) Time course of average $I_{\mathrm{d}} / g_{\mathrm{m}}$ at $V_{\mathrm{d}}$ of $0.1 \mathrm{~V}$ and $V_{\mathrm{g}}$ of $0 \mathrm{~V}$ for MBP-V $\mathrm{V}_{\mathrm{L}}$ modified G-FETs at various concentration of BGP-C7 and constant concentration of Trx-BAP-V $V_{H}$ of 1 $\mu \mathrm{g} / \mathrm{mL}$. 


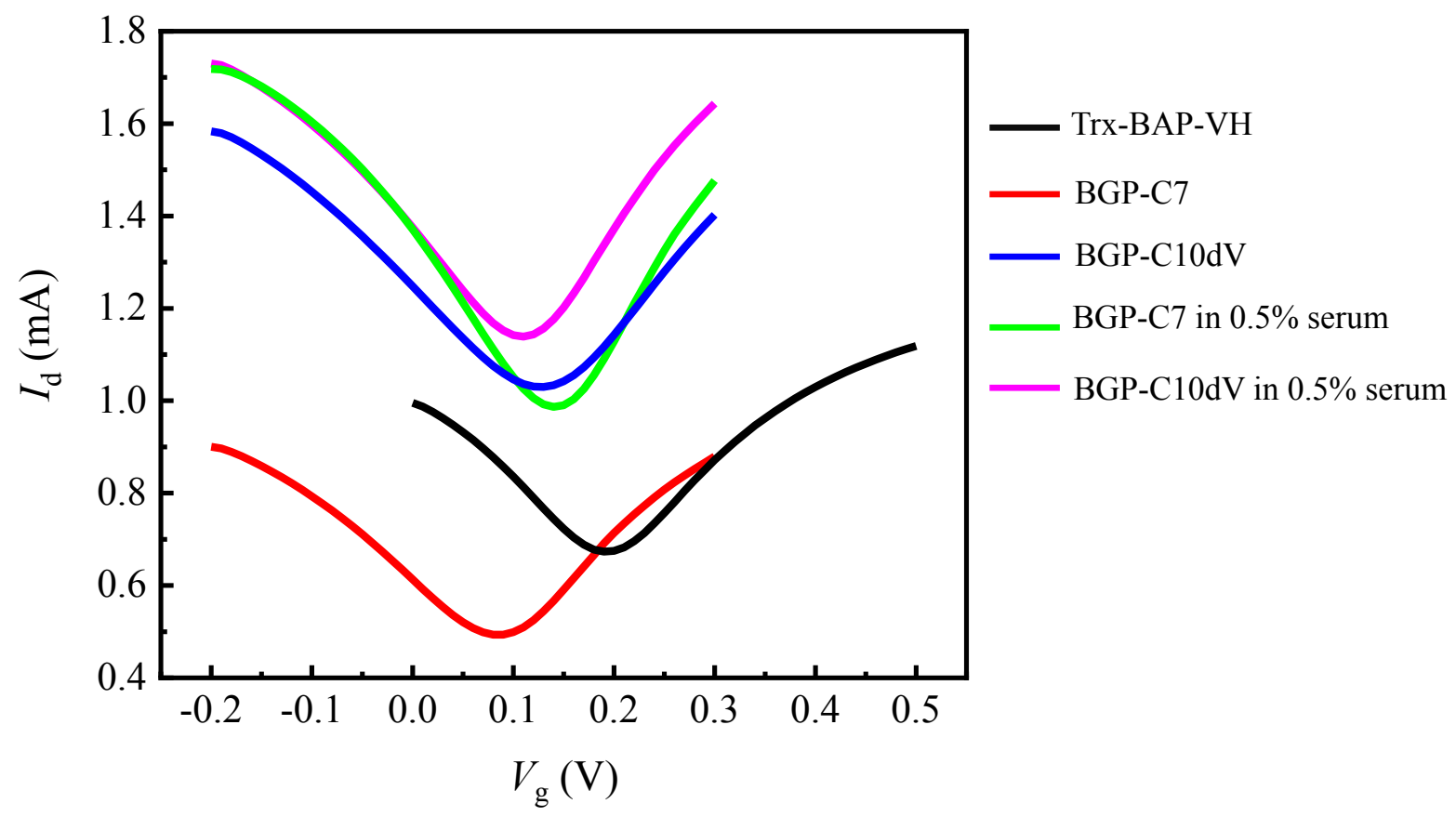

Figure S3. Transport characteristics of G-FETs prepared for each measurement. Transport characteristics of G-FETs combined with APP- $\mathrm{V}_{\mathrm{H}}$ used for the detection of BGP-C7 in PB (red), BGP-C10dV in PB (blue), BGP-C7 in $0.5 \%$ human serum (green) and BGP-C10dV in $0.5 \%$ human serum (magenta), as well as that of G-FET with Trx-BAP- $\mathrm{V}_{\mathrm{H}}$ (black) as in Figure S2A are shown. 